RESEARCH NOTE

\title{
Study on Detectability of Signals by Utilizing Differences in Their Amplitude Modulation
}

\author{
Yuta Yano, Ryota Miyauchi, Masashi Unoki and Masato Akagi
School of Information Science, Japan Advanced Institute of Science and Technology
1-1 Asahidai, Nomi, Ishikawa 923-1292, Japan
E-mail: \{s0810064, ryota, unoki, akagi\}@jaist.ac.jp

\begin{abstract}
Auditory search experiments were conducted to investigate the detectability of target signals with regard to movements in temporal envelopes. The movements in the temporal envelopes of signals were defined as the mean value of slopes, i.e., the first-order approximation of the temporal envelopes. The movements were systematically controlled by band-pass filtering on the modulation spectrum of the signals. The movements of background signals in each target signal were set to be different from the movements between target and background signals. Stimuli were composed of different temporal envelopes with a noise carrier. 1/2-octave band-noise as a carrier was used in which the center frequency of the carrier was 1380 Hz. Participants were required to determine whether they heard the target signal with the background signals. The results obtained from auditory search experiments demonstrated that the detectability of each target signal could be improved when the movements of the target and background signals differed. The results revealed that the difference in the movements of the signals affected the detectability of the signals.
\end{abstract}

Keywords: amplitude modulation, auditory search, alarm detection, movements in temporal envelope

\section{Introduction}

Audible alarm signals are used to attract the attention of people in many everyday activities, e.g., the sounds of fire alarms in emergencies and the beeps and melodic sounds of electronic products that alert us of providing start and end times [1]. These signals must be perceived accurately and efficiently by everyone. Alarm signals with many different stimulus signatures have been studied for this purpose to check if they can be adequately perceived, e.g., by Mizunami et al. [2]. There are, however, cases where alarm signals cannot be correctly perceived in real environments because they are masked or partially masked by background noise, and only the person they are intended for knows when and what events have occurred. Therefore, it is important to present alarm signals in such a way that they can be correctly detected in any environment. Here, we can replace the detectability of target signals with auditory search tasks.

Asemi et al. [3], in a study that had a close relationship to ours, carried out research on auditory search tasks. Narrow-band noise and pure tones were used and temporal fluctuations in the target were controlled in their experiments as cues to investigating improvements in the detectability of target signals. Their results indicated that temporal fluctuations in the target against background signals played a role in improving detectability.

Based on their results, Kusaba et al. investigated how much temporal fluctuations between the target and background signals could improve detectability of the target signal in auditory search experiments [4]. Their results suggested that less similarity in temporal fluctuations between the target and background signals could improve the detectability of the target in noisy environments. However, as they used directed narrow-band noise as stimuli in their experiments, the temporal fluctuations were not controlled.

We defined movements in our previous study and carried out auditory search experiments by using controlled movements. The results suggested that the detectability of target signals was improved as the difference in the movements increased. This tendency constantly appeared when the movement of the target signal was greater than that of the background signal [5]. However, this experiment had two factors for movement and the carrier frequency, so that we could not investigate the factor for movement only.

We investigated the relationship between movements and the parameters of the bandpass filter in this study. Based on this investigation, we generated stimuli where the values for the difference in the movements of the target and background signals were 

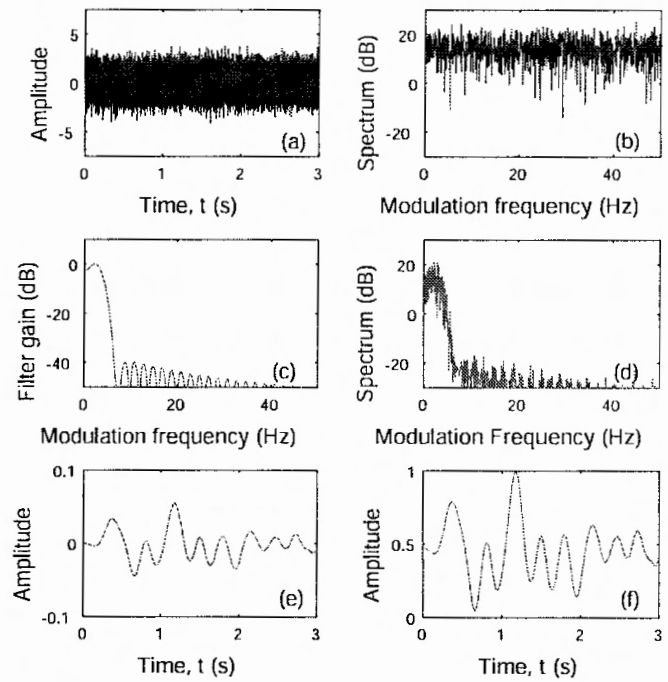

Fig, 1 Examples of generation of stimuli: (a) white noise, (b) modulation spectrum of white noise, (c) bandpass (BP) filter on modulation spectrum, (d) modulation spectrum of BP-filtered noise, (e) amplitude envelope, and (f) normalized amplitude envelope

evenly distributed, and we carried out an auditory search experiment. We considered that the detectability of a signal was affected in this way by utilizing movements of the signals and determined whether we would be able to control the detectability of signals through movements.

\section{Movements in Temporal Envelopes}

We defined movement in the temporal envelope of a signal as the mean value of slopes of the temporal envelope [5].

$$
v=\frac{A_{0}}{N-1} \sum_{n=2}^{N} \frac{\left|P_{n}-P_{n-1}\right|}{T_{n}-T_{n-1}}
$$

where $P_{n}$ represents the values of peaks/dips in temporal envelopes, $T_{n}$ is the time, and $N$ is the number of peaks/dips. Here, $A_{0}$ is a normalized factor and this is set to be the output reference voltage (using the TDT System III and SANSUI AU- $\alpha$ 907MR Integrated Amplifier). The $A_{0}$ in this experiment was $88 \mathrm{mV}$. Therefore, $v$ was in millivolts per second.

We systematically controlled the movements defined above with by bandpass (BP) filtering on the modulation spectrum as follows. We assumed that signals could be expressed by multiplying the temporal envelope with the carrier. Here, band-noise was used as the carrier.

Figure 1 shows an example of the generation of stimuli that we used in the auditory search experiment. White noise was used to generate the stimuli, as shown in Fig. 1(a). Figure 1(b) shows the modulation spectrum of (a). Figure 1(c) shows the characteristics of the BP filter at the modulation frequency. We changed the bandwidth and CF of the BP filter to control the movements of signals. The filter shown in Fig. 1(c) has the bandwidth of $2.5 \mathrm{~Hz}$ at the $\mathrm{CF}$ of 2 $\mathrm{Hz}$. The processed modulation spectrum was obtained by $\mathrm{BP}$ filtering on the modulation spectrum shown in Fig. 1(d). The processed amplitude envelope was then obtained from the inverse Fourier transform shown in Fig. 1(e). This was normalized by the averaged value for amplitude to be \pm 0.5 . Stimuli were generated by multiplying the temporal envelopes with the carriers. The rising and falling edges of the stimuli were created as a 30 -ms raised-cosine.

We systematically controlled the envelopes shown in Fig. 1(f) by using Eq. (1) to control movements in the temporal envelopes. As in the above, various movements were generated by systematically controlling their modulation spectrum using the BP filter.

\subsection{Relationship between movements and parame-} ters of BP filter

We investigated the detectability of signals based on differences in the movements of target and background signals in this study. To do this, we needed to generate stimuli where the differences in movements became equal. We calculated the values for the movements of temporal envelopes by BP filtering, which used various CFs and bandwidths for the $\mathrm{BP}$ filter, to investigate the relationship between the movements and parameters of the filter.

Figure 2 plots the distribution in the values of movements obtained from each BP filtering on the modulation spectrum. We found from this figure that the movements tended to increase commensurately as the CF of the filter increased. Therefore, we could systematically control the movements by varying the parameters of the filter. However, there was variability in the values of movements obtained from the same $\mathrm{BP}$ filter, because the values were affected by the differences of the original temporal envelopes. Thus, we pegged the mean of values for movements at a movement obtained from BP filtering on the modulation spectrum.

Based on the results, we set the CF and bandwidth of the BP filter for using in following auditory search experiment in such a way that each value for different movements was $220 \mathrm{mV} / \mathrm{s}$. Table 1 summarizes the relationship between the movements and parameters of the BP filter.

\section{Auditory Search Experiment}

\subsection{Aim and stimuli}

We carried out an auditory search experiment for the target and background signals with different movements added to investigate the detectability of the target with regard to movements in temporal envelopes. 
Table 1 Relationship between movements and parameters of BP filter

\begin{tabular}{|c|c||c|}
\hline $\mathrm{CF}(\mathrm{Hz})$ & bandwidth $(\mathrm{Hz})$ & movements $(\mathrm{mV} / \mathrm{s})$ \\
\hline \hline 2.00 & 1.0 & 88 \\
\hline 4.75 & 1.5 & 308 \\
\hline 9.25 & 1.5 & 528 \\
\hline 11.50 & 1.0 & 748 \\
\hline 14.25 & 1.5 & 968 \\
\hline 16.25 & 1.5 & 1188 \\
\hline 20.00 & 1.0 & 1408 \\
\hline
\end{tabular}

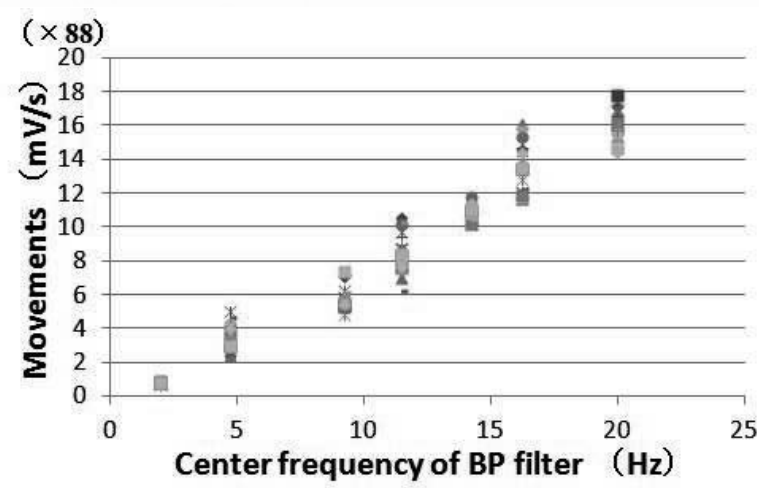

Fig. 2 Distribution in movements obtained from modulation spectrum controlled by BP filtering

The movements in temporal envelopes that we used in the experiment were $44,154,264,374,484,594$, and $704 \mathrm{mV} / \mathrm{s}$. These values were obtained from Fig. 2 and Tab. 1 in which the movements in temporal envelopes were evenly arranged by controlling center frequency of the BP filtering. Three of them - -264 , 374 , and $484 \mathrm{mV} / \mathrm{s}$ - were used as target signals. The movements of background signals in each target were set to be different from the movements between target and background signals. When the movement of the target was $264 \mathrm{mV} / \mathrm{s}$, the movements of the background were $44,154,374$, and $484 \mathrm{mV} / \mathrm{s}$. When the movement of the target was $374 \mathrm{mV} / \mathrm{s}$, the movements of the background were $154,264,484$, and $594 \mathrm{mV} / \mathrm{s}$. When the movement of the target was $484 \mathrm{mV} / \mathrm{s}$, the movements of the background were $264,374,594$, and $704 \mathrm{mV} / \mathrm{s}$. Stimuli were composed of different temporal envelopes with a noise carrier. 1/2-octave bandnoise as a carrier was used in which the center frequency of the carrier was $1380 \mathrm{~Hz}$.

\subsection{Procedure and apparatus}

There were two kinds of assessments in the auditory search experiment, i.e., positive and negative. Stimuli as the target and background signals were simultaneously presented to the participants via headphones in the positive assessments. Stimuli as background signals and band-noise in which movement was not controlled were simultaneously presented to the participants in the negative assessments. The partici-

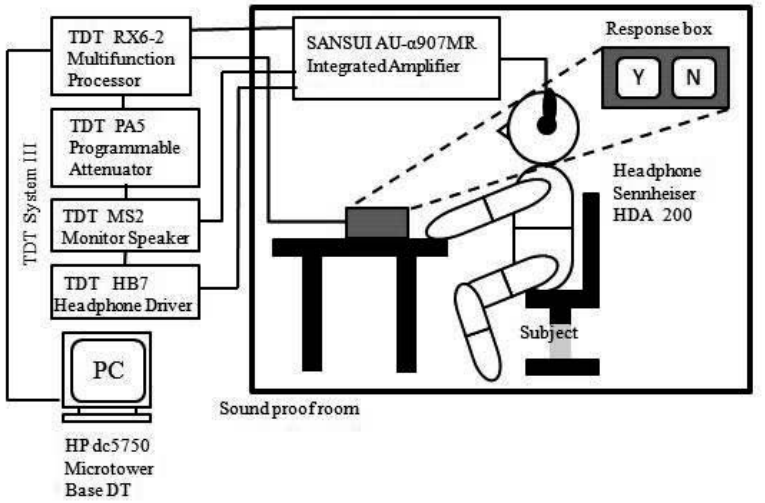

Fig. 3 Experimental apparatus

pants were required to determine whether they heard the target signal with the background signal and to press a "Y (yes)" or "N (no)" button on the response box. Before the participants started the sessions in this experiment, a target signal was repetitively presented to them so that they could learn the target signal that would be presented in the session. There were a total of 240 trials in the session (three target signals $x$ four background signals $x$ two assessments (positive and negative) $\times$ ten repetitions). These trials were randomly presented.

Figure 3 has a diagram of the apparatus we used in the experiment, which was carried out in a soundproof room. Stimuli were generated by Tucker-Davis Technologies (TDT) System III and presented to each subject through an amplifier (SANSUI, AU- $\alpha$ ) and headphones (Sennheiser HDA200). Subjects' responses were recorded through the response box.

\section{Results}

We obtained the detectability of the target signal, i.e., the discriminability index, $d^{\prime}$, from the results of the assessments done by the participants. There were four classifications of the results for the assessments: Hit, (they pressed "Y" when there was a target), Miss (they pressed "N" even though there was a target), False Alarm (they pressed "Y" even though there was no target), Correct Rejection (they pressed " $N$ " when there was no target). Based on the proportions of these types of trials, we obtained numerical estimates of detectability with the discriminability index $\left(d^{\prime}\right)$. Greater values for $d^{\prime}$ indicated that the target signal was easily detected.

Figure 4 plots the detectability of the target signal obtained by using the difference between movements in the temporal envelopes of the target and background signals. The movement in target signals was $264 \mathrm{mV} / \mathrm{s}$. Figure 5 plots the detectability of target signals when the movement of the target was 374 $\mathrm{mV} / \mathrm{s}$. Figure 6 plots the detectability of target signals when the movement of the target was $484 \mathrm{mV} / \mathrm{s}$.

The results demonstrated that the detectability of target signals was improved when the movements of the target and background signals differed. They also 


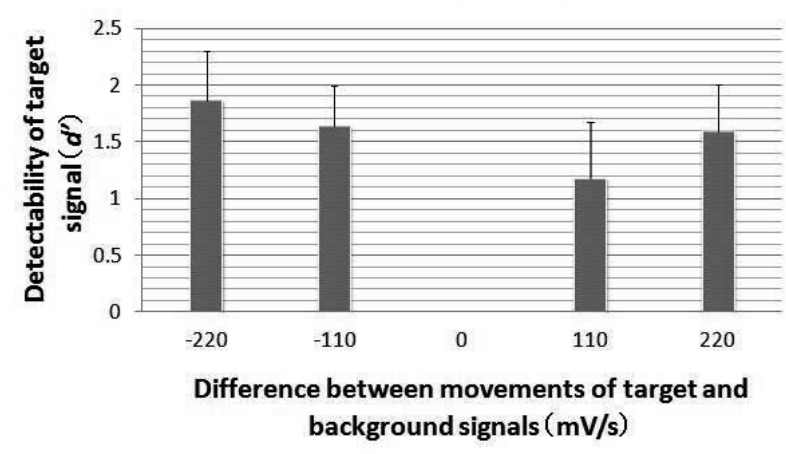

Fig. 4 Detectability of target signals obtained by using the differences in movements (movement was 264 $\mathrm{mV} / \mathrm{s}$ )

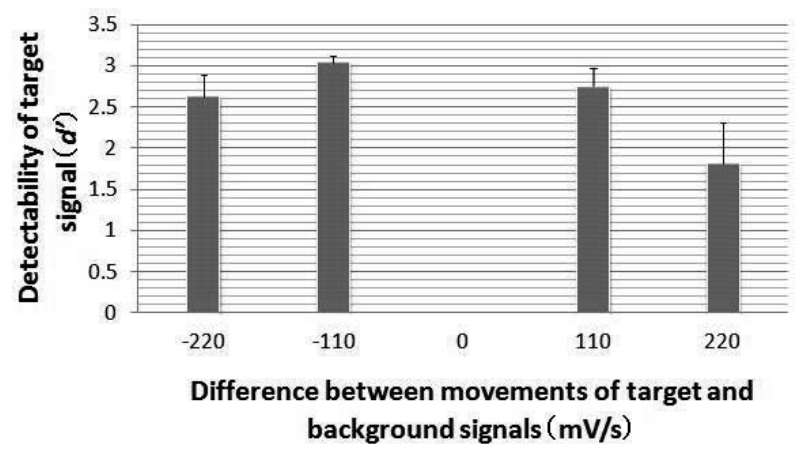

Fig. 5 Detectability of target signals obtained by using the differences in movements (movement was 374 $\mathrm{mV} / \mathrm{s}$ )

suggested that the target signals were easily detected by applying different movements to the target and background signals.

We used band-noise that had the same CF and bandwidth as the carrier of the target and background signals in this experiment. The target and background signals passed through the same auditory filter under these conditions. This indicated that these two signals were decomposed into one component as one sound. Thus, it was extremely difficult to separate the two signals. However, under such difficult conditions, these results suggest that we could perceptually separate two signals and detect a target signal by perceiving the amplitude modulation of the signals.

We obtained results in our previous study where the detectability of target signals was improved as the difference in movements increased. However, there was little to distinguish the detectability of signals in these results, in comparison with cases where the difference in movements was $110 \mathrm{mV} / \mathrm{s}$ and cases where the difference in movements was $220 \mathrm{mV} / \mathrm{s}$. This was attributed to the fact that we could completely separate the two signals when the difference in movements between the target and background signals was only $110 \mathrm{mV} / \mathrm{s}$, so that detectability remained constant even if we used further differences. We did not examine perceptual differences in movement in this experiment. Therefore, we need to set experimental conditions in investigations that are not based on phys-

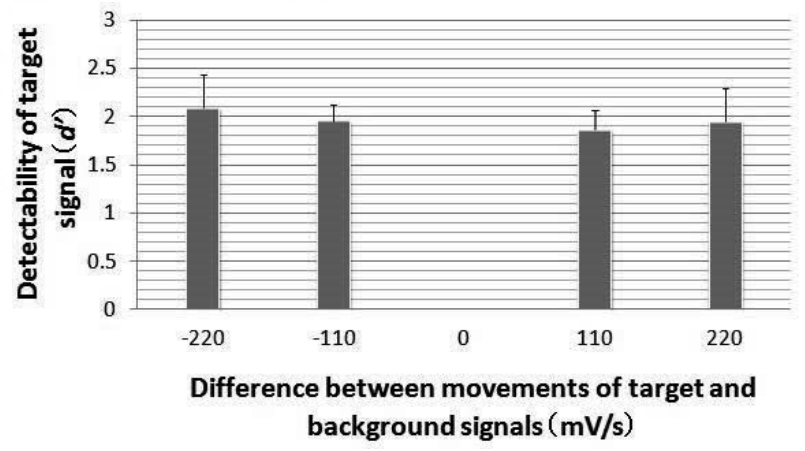

Fig. 6 Detectability of target signals obtained by using the differences in movements (movement was 484 $\mathrm{mV} / \mathrm{s}$ )

ical but perceptual differences in movements to find how differences in movements affect the detectability of target signals.

\section{Conclusion}

We defined movements in the temporal envelopes of signals and proposed a method of controlling these movements. The auditory search experiments were carried out by using target and background signals with different movements. The results demonstrated that the detectability of target signals could be improved when the movements of the target and background signals differed. This indicated that the amplitude modulation of signals was closely related to the detectability of target signals. We discovered that the detectability of signals could be controlled by utilizing differences in movements.

\section{Acknowledgments}

This work was supported by a Grant-in-Aid for Challenging Exploratory Research (No. 24653211).

\section{References}

[1] JIS S 0013: Guidelines for the elderly and people with disabilities - Auditory signals on consumer products, 2002.

[2] T. Mizunami, K. Kurakata, H. Shimosako and K. Matsushita: Further examination of ON/OFF temporal patterns of auditory signals (completion signals and attention signal) recommended in JIS S 0013, J. Ergonomics, Vol. 40, No. 5, pp. 264 271, 2005.

[3] N. Asemi, Y. Sugita and Y. Suzuki: Auditory search asymmetry between normal Japanese speech sounds and time-reversed speech sounds distributed on the frontalhorizontal plane, Acoust. Sci. \& Tech., Vol. 24, No. 3, pp. $1451.47,2003$.

[4] M. Kusaba, M. Unoki and M. Akagi: A Study on detectability of target signal in background noise by utilizing similarity of temporal envelopes in auditory search, Proc. NCSP'08, pp. 13-16, 2008. 
[5] Y. Yano, R. Miyauchi, M. Unoki and M. Akagi: Study on detectability of target signal by utilizing differences between movements in temporal envelopes of target and background signals, Proc. NCSP'11, pp. 231 234, 2011.

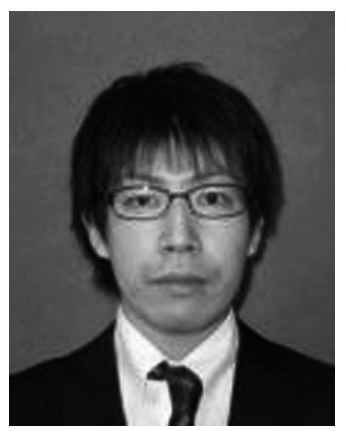

Yuta Yano received his B.E. from Ehime University in 2007. He received his M.S. from the Japan Advanced Institute of Science and Technology (JAIST) in 2012. His main research interest is speech signal processing. He currently works in the EHIME COMPUTING CENTER.

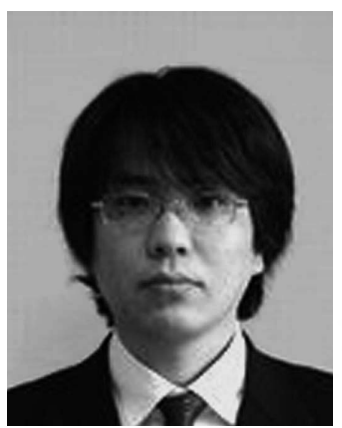

Ryota Miyauchi received his Bachelor's and Master's degree and Ph.D from Kyushu Institute of Design in 1998, 2000, and 2005 , respectively. He joined $\mathrm{Re}-$ search Institute of Electrical Communication of Tohoku University as Assistant Professor. Presently, he is an Assistant Professor of Japan Advanced Institute of Science and Technology. His current research interests include spatiotemporal perception and multi sensory integration based on hearing.

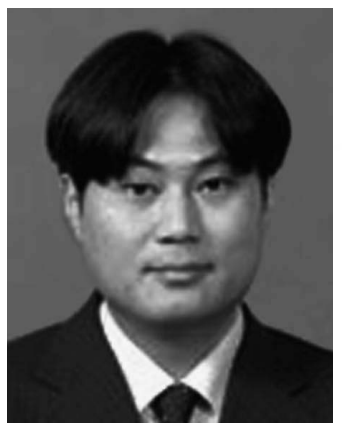

Masashi Unoki received his M.S. and Ph.D. (Information Science) from the Japan Advanced Institute of Science and Technology (JAIST) in 1996 and 1999. His main research interests are in auditory motivated signal processing and the modeling of auditory systems. He was a Japan Society for the Promotion of Science (JSPS) research fellow from 1998 to 2001. He was associated with the ATR Human Information Processing Laboratories as a visiting researcher from 1999-2000, and he was a visiting research associate at the Centre for the Neural Basis of Hearing (CNBH) in the Department of Physiology at the University of Cambridge from 2000 to 2001. He has been on the faculty of the School of Information Science at JAIST since 2001 and is now an Associate Professor. He is a member of the Research Institute of Signal Processing (RISP), the Institute of Electronics, Information and Communication Engineers (IEICE) of Japan, and the Acoustical Society of America (ASA). He is also a member of the Acoustical Society of Japan (ASJ), and the International Speech Communication Association (ISCA). Dr. Unoki received the Sato Prize from the ASJ in 1999 and 2010 for an Outstanding Paper and the Yamashita Taro "Young Researcher" Prize from the Yamashita Taro Research Foundation in 2005.

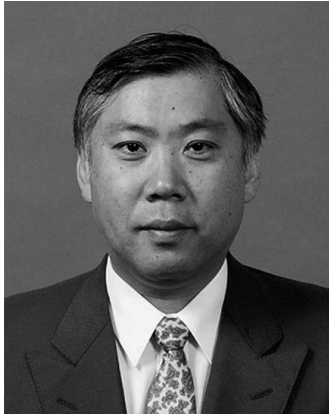

Masato Akagi received his B.E. from Nagoya Institute of Technology in 1979 , and his M.E. and Ph.D. Eng. from the Tokyo Institute of Technology in 1981 and 1984. He joined the Electrical Communication Laboratories of Nippon Telegraph and Telephone Corporation (NTT) in 1984. From 1986 to 1990 , he worked at the ATR Auditory and Visual Perception Research Laboratories. Since 1992 he has been on the faculty of the School of Information Science of the Japan Advanced Institute of Science and Technology (JAIST) and is now a full Professor. His research interests include speech perception, the modeling of speech perception mechanisms in human beings, and the signal processing of speech. During 1998, he was associated with the Research Laboratories of Electronics at MIT as a visiting researcher, and in 1993 he studied at the Institute of Phonetics Science at the University of Amsterdam. He is a member of the Institute of Electronics, Information and Communication Engineers (IEICE) of Japan, the Acoustical Society of Japan (ASJ), the Institute of Electrical and Electronic Engineering (IEEE), the Acoustical Society of America (ASA), and the International Speech Communication Association (ISCA). Dr. Akagi received the IEICE Excellent Paper Award from the IEICE in 1987, the Best Paper Award from the Research Institute of Signal Processing in 2009, and the Sato Prize for Outstanding Papers from the ASJ in 1998, 2005, 2010 and 2011. Professor Akagi is currently the president of the ASJ.

(Received May 13, 2012; revised August 18, 2012) 